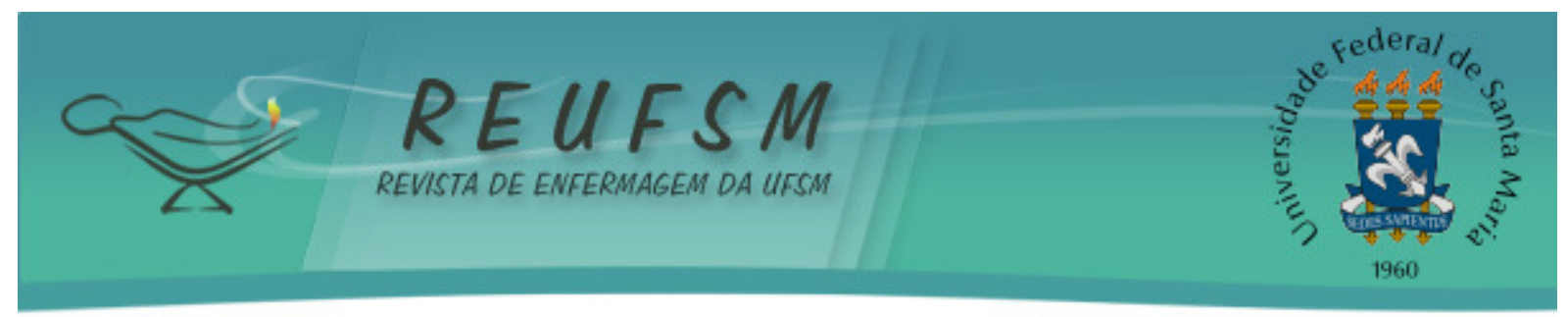

RELATO DE EXPERIÊNCIA

\title{
APRENDIZAJE BASADO EN PROBLEMAS CON ESCENARIOS SIMULADOS: UN MODELO PEDAGÓGICO AUTODIRIGIDO EN ENFERMERÍA
}

\author{
PROBLEM-BASED LEARNING WITH SIMULATED SCENARIOS: A SELF-TEACHING MODEL IN \\ NURSING
}

\section{APRENDIZAGEM BASEADA EM PROBLEMAS COM CENÁRIOS SIMULADOS: UM MODELO PEDAGÓGICO AUTO-DIRIGIDO EM ENFERMAGEM}

Doi: $10.5902 / 2179769210863$

José Luis Díaz Agea ${ }^{1}$

César Leal Costa ${ }^{2}$

RESUMEN: Objetivo: este trabajo tiene como objetivo presentar un modelo de fusión de dos metodologías de aprendizaje activo como son el Aprendizaje basado en problemas y la simulación clínica en enfermería. Este modelo está fundamentado en la experiencia combinada puesta en marcha de manera completa en $4^{\circ}$ de grado de enfermería en la Universidad Católica San Antonio de Murcia (España). Método: en grupos de 18 alumnos y subgrupos de 3 se eligen las situaciones clínicas que habrán de trabajar. Los alumnos realizan una lluvia de ideas y marcan los objetivos de aprendizaje. Diseñan el escenario, lo representan en la sala de simulación clínica y lo discuten llevando el peso del Debriefing y fundamentando su análisis en evidencias científicas. Resultados: se ha observado una mayor implicación del alumnado y una mayor participación en el Debriefing. Conclusiones: se estima que el aprendizaje se refuerza con la suma de ambas metodologías.

Descriptores: Aprendizaje basado en problemas; Simulación por computador; Aprendizaje; Enfermería.

ABSTRACT: Objective: the purpose of this paper is to present a model of fusion of two active learning methods, namely the Problem-based Learning and the Clinical Simulation in Nursing. This model is based on merged experience, thoroughly implemented in the 4th year of Nursing at the Catholic University San Antonio of Murcia (Spain). Method: the activities are carried out in groups of 18 students and subgroups of three, in which the clinical cases to be worked on are chosen. Students brainstorm so as to reach the learning objectives. They describe the scenario, represent it in the simulation room and discuss it using Debriefing as a foundation and performing an analysis based on scientific evidence. Results: there has been a greater involvement of the students and greater participation in the Debriefing. Conclusion: it can be noticed that learning is reinforced by the addition of both methodologies.

Descriptors: Problem-based learning; Computer Simulation; Learning; Nursing.

RESUMO: Objetivo: este artigo tem como proposta apresentar um modelo de fusão de dois métodos de aprendizagem ativos, tais como Problem Based Learning e simulação clínica em enfermagem. Este modelo está fundamentado na experiência combinada,

\footnotetext{
1 Doctor en Ciencias Socio-sanitarias por la UCAM y enfermero. Profesor Contratado Doctor. Instructor en Simulación Clínica. Facultad de Enfermería. Universidad Católica de Murcia. España. E-mail: jluis@ucam.edu

2 Doctor en Psicología por la Universidad Miguel Hernández y enfermero. Profesor Contratado Doctor. Instructor en Simulación Clínica. Facultad de Enfermería. Universidad Católica de Murcia. España. E-mail: cleal@ucam.edu
} 


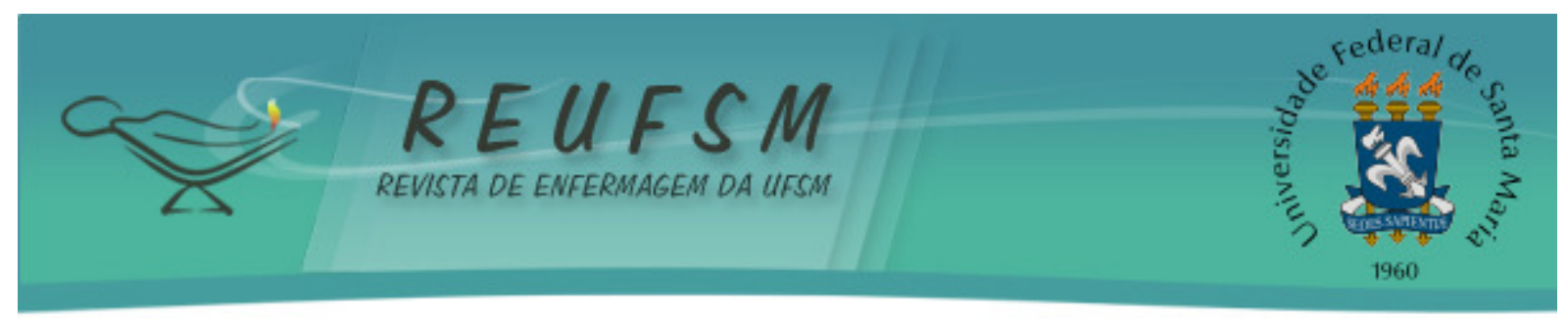

implementada plenamente no $4^{\circ}$ ano de enfermagem da Universidade Católica San Antonio de Murcia (Espanha). Método: as atividades são realizadas em grupos de 18 alunos e subgrupos de três, em que se elegem as situações clínicas que terão que trabalhar. Os alunos realizam uma chuva de ideias e alcançam os objetivos de apredizagem. Descrevem o cenário, o representam na sala de simulação clínica e o discutem tendo como base o Debriefing e fundamentando sua análise em evidências científicas. Resultados: observa-se uma maior envolvimento dos alunos e uma maior participação no Debriefing. Conclusão: percebe-se que a aprendizagem é reforçada com a soma de ambas as metodologias.

Descritores: Aprendizagem baseada em problemas; Simulação por Computador; Aprendizagem; Enfermagem.

\section{INTRODUCCIÓN}

En el presente artículo se presenta un modelo de fusión del Aprendizaje Basado en Problemas (ABP) y la simulación clínica llevado a cabo en la unidad de simulación de la Facultad de Enfermería de la Universidad Católica San Antonio de Murcia (UCAM). Tanto el ABP como la simulación clínica son dos metodologías docentes centradas en el aprendizaje del alumno como sujeto activo en la adquisición de habilidades, conocimientos y actitudes. ${ }^{1}$ Ambas experiencias educativas se han llevado a cabo por separado en el itinerario formativo del pregrado de enfermería de algunas universidades y centros sanitarios españoles. ${ }^{2,3}$

En la UCAM se ha utilizado el ABP en la Facultad de Enfermería ${ }^{4}$ en una experiencia pedagógica acorde a las directrices europeas de enseñanza superior. ${ }^{5}$ Por otra parte, la simulación clínica con escenarios complejos se ha implantado como parte de la formación de los alumnos de grado en enfermería, constituyéndose además como una herramienta de evaluación de competencias profesionales. ${ }^{6}$

Recientemente $^{7}$ se han descrito las ventajas de vincular ambas metodologías justificando convenientemente su uso en la adquisición de un mayor nivel de competencias. En el Practicum VI de $4^{\circ}$ de grado en enfermería de la UCAM se han fusionado ambas metodologías (ABP y simulación), lo que ha posibilitado conjuntar las ventajas de ambas formas de aprendizaje. En un estudio reciente ${ }^{8}$ se ha demostrado que los alumnos de último curso de enfermería que utilizan ambas metodologías han obtenido mejores calificaciones que la simulación clínica por sí sola, lo que podría considerarse como una mejora en la adquisición de competencias y en la experiencia de aprendizaje. El entrenamiento en habilidades humanas se puede incrementar propiciando escenarios donde el aprendizaje autoadministrado del alumno condiciona una mayor experiencia de reflexión, así lo pone de manifiesto un estudio ${ }^{9}$ en el que se mejoraron las actitudes de los alumnos de enfermería, mediante ABP y simulación.

La simulación clínica con escenarios complejos y de alta fidelidad también se va abriendo camino en el ámbito universitario brasileño, ${ }^{10,11}$ aunque el alto precio de los equipos es la principal limitación a la implantación de esta metodología. ${ }^{11,12}$ No obstante, lo que propicia el aprendizaje no es la tecnología en sí, sino que la discusión posterior a la acción simulada (Debriefing) es crucial para que se genere ese diálogo necesario entre la teoría y la práctica. El que se produce aumenta las posibilidades de fijar conocimientos asociados a la acción experimentada. ${ }^{13}$

El aprendizaje es un proceso continuo que se fundamenta en la experiencia y que implica una transacción entre personas y el ambiente. ${ }^{14}$ Con los ingredientes del autoaprendizaje, la motivación, la experiencia vivida, el feedback y la reflexión se puede justificar que la integración de ABP y simulación clínica resulta metodológicamente posible y llevado a la práctica es una experiencia educativa motivadora para el alumno y el 


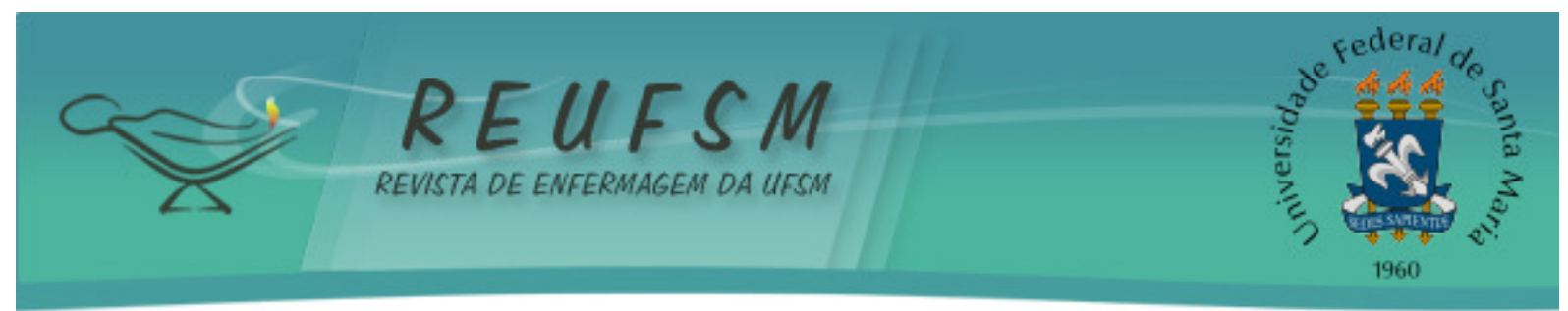

instructor. No hay que olvidar que, como se ha puesto de manifiesto en un reciente estudio, ${ }^{15}$ se debe incentivar la formación de profesionales de enfermería críticos y reflexivos, lo que redundará en una mejora de los cuidados prestados a la comunidad.

Como objetivos nos planteamos justificar la pertinencia de la fusión de metodologías de aprendizaje activo en enfermería como la simulación clínica avanzada y el Aprendizaje Basado en Problemas, así como describir el modelo educativo del Practicum VI de la Universidad Católica San Antonio de Murcia que integra ambas metodologías.

\section{METODOLOGÍA}

Tanto el ABP como la simulación clínica con escenarios complejos están suficientemente descritos por la bibliografía ${ }^{16-19}$ y tienen una larga trayectoria en la enseñanza universitaria en ciencias de la salud a nivel internacional. El modelo de simulación que aquí se presenta se sustenta en la metodología enfermera y el proceso de enfermería utilizando el lenguaje estandarizado de la North American Nursing Diagnosis Association (NANDA) ${ }^{20}$ la clasificación de resultados $(N O C)^{21}$ y la clasificación de intervenciones (NIC). ${ }^{22}$ Por lo que metodológicamente supone una aproximación realista a la experiencia clínica estandarizada que se propugna en la disciplina de enfermería.

Este modelo se lleva a cabo con la puesta en práctica de escenarios clínicos de muy diversa índole seleccionados por los propios alumnos en grupos reducidos. La discusión posterior de dichos escenarios se basa en búsquedas de evidencia científica llevadas a cabo por los propios estudiantes que conforman el subgrupo (tres alumnos) que ha diseñado el escenario, lo ha escenificado en la sala de simulación (Figura 1) y ha llevado el peso de la discusión ejerciendo de moderadores y facilitadores de la misma. El instructor de simulación pasa a un segundo plano salvo que tenga que reconducir la situación, manejar el software y el equipo de simulación avanzada y videograbación o fomentar el análisis y la reflexión.

En un primer momento se realiza un seminario donde se explica la metodología que se va a utilizar y se constituyen los subgrupos de trabajo. Se presentan una batería de situaciones extraídas de la realidad. Las situaciones se basan en el cuaderno de competencias que tienen a su disposición los alumnos y que se elabora como un documento de consenso ${ }^{23}$ para las prácticas clínicas junto con la planificación de la asignatura correspondiente. 

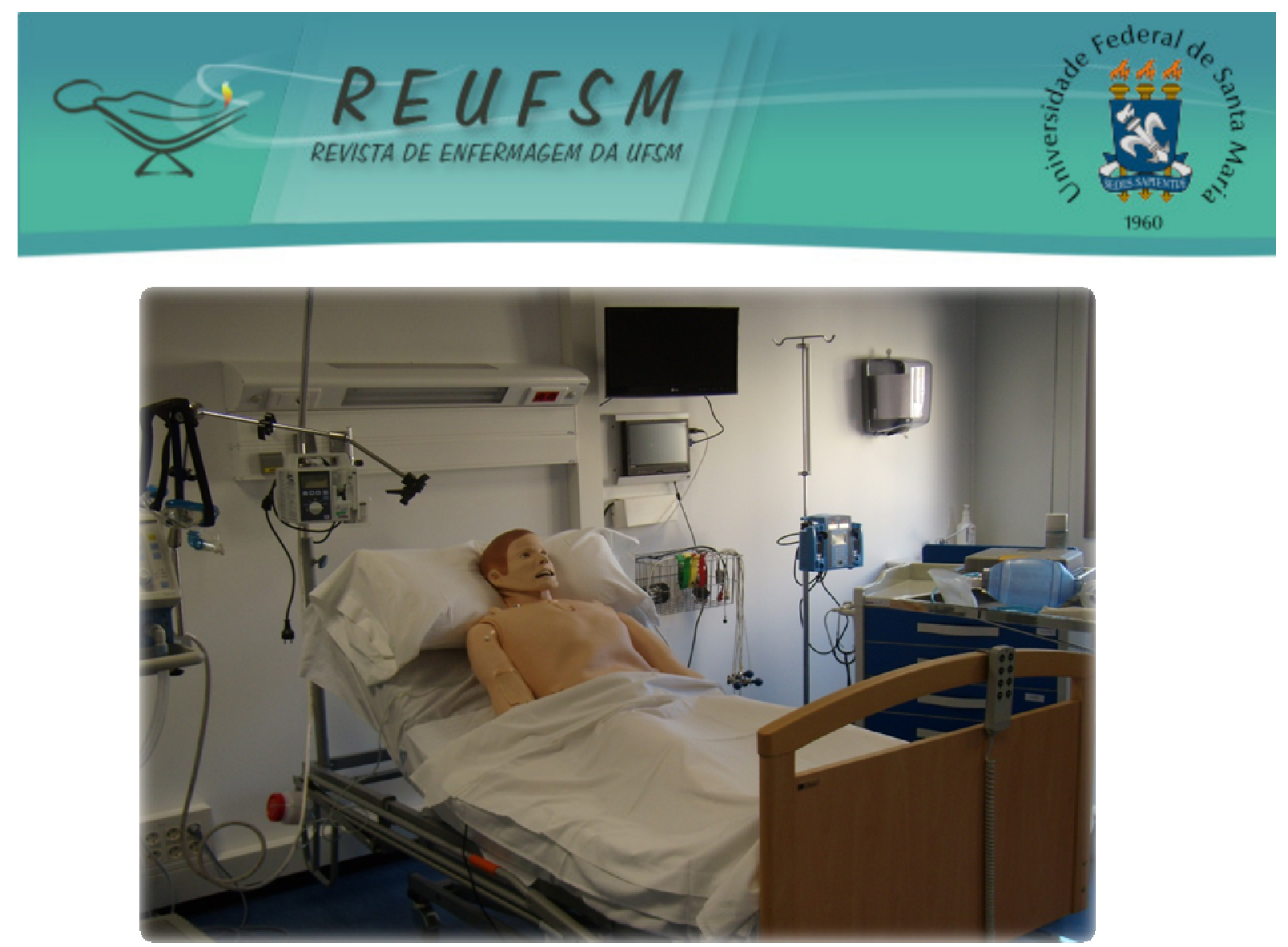

Figura 1. Sala de simulación de la Facultad de Enfermería de la UCAM.

Una vez escogidas las situaciones se establece un debate entre el grupo y con la técnica de la lluvia de ideas (brainstorming) se establecen varios parámetros desde lo general hasta lo concreto: qué sabemos de la situación, qué desconocemos, qué queremos saber y dónde podemos encontrar la información. De manera que del brainstorming emanan los objetivos de aprendizaje y además el grupo ofrece sugerencias e ideas para el diseño de cada uno de los escenarios de simulación que se trabajarán en la siguiente sesión. El instructor reconduce la situación si ésta se desvía y ejerce un papel de moderador, pero no resulta conveniente que adopte un papel directivo. ${ }^{24}$ Trascurrida una semana, y con un trabajo grupal de por medio, los estudiantes realizan la simulación clínica en las salas de simulación con los escenarios seleccionados y posteriormente se discuten en el Debriefing (que consiste en discutir lo experimentado en base a identificar los puntos débiles y fuertes, así como las propuestas de mejora, siempre desde el punto de vista de los participantes, los observadores y por último el instructor).

\section{RESULTADOS}

\section{Planificación de las sesiones de simulación con Aprendizaje Basado en Problemas}

El $A B P$ se integra con la simulación en una estructura dinámica y secuencial compuesta por seis sesiones en las que se eligen los casos, se establecen los objetivos de aprendizaje, se diseña una estrategia de búsqueda y se discute el conocimiento previo. Posteriormente se escenifica el caso y se discute con evidencias. En la Figura 2 exponemos la planificación de las diferentes sesiones. 

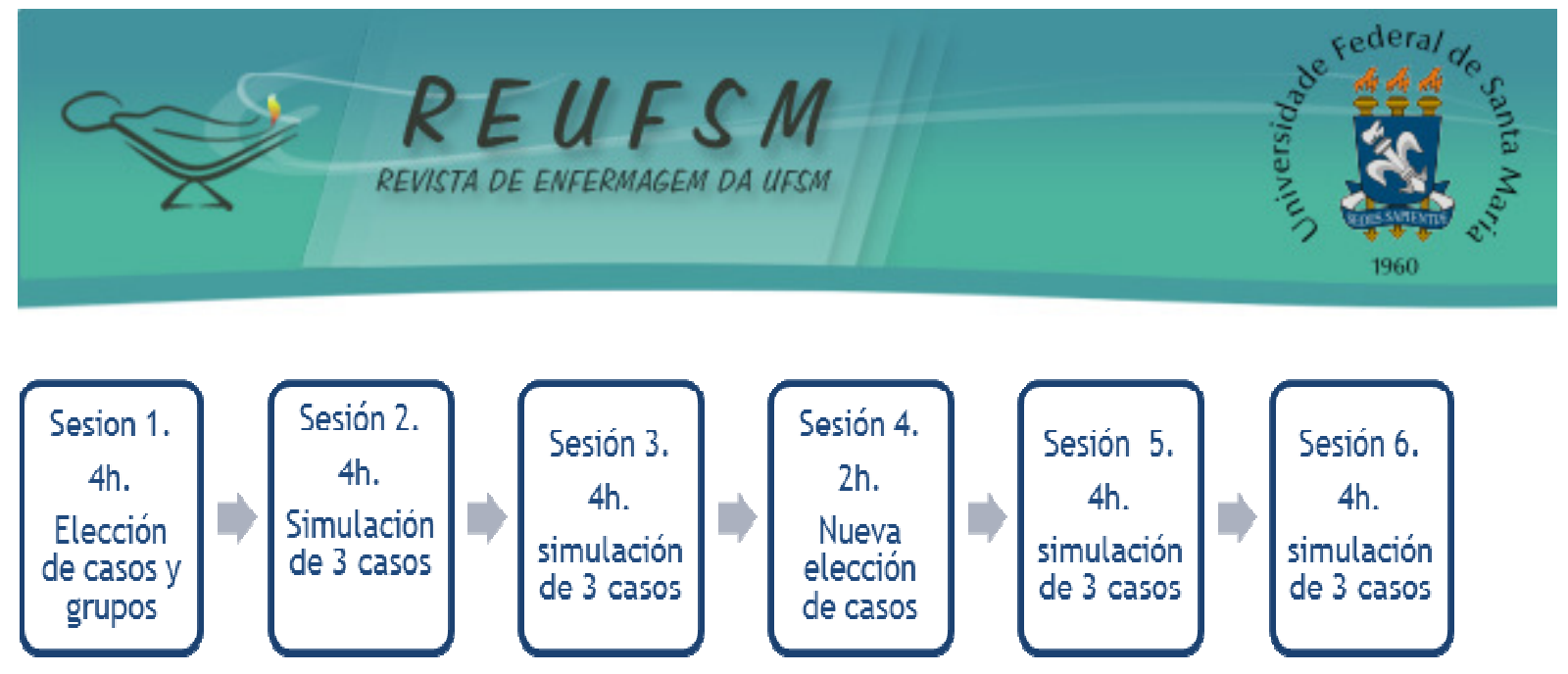

Figura 2. Planificación de las sesiones. Elaboración propia.

\section{Desarrollo de las sesiones. Papel del alumno y del tutor}

En la primera sesión se ofrecen una batería de posibles temáticas para elaborar escenarios de acuerdo al cuaderno de competencias del Practicum VI. Son posibles escenarios extraídos de la realidad y en consonancia con las unidades en las que los alumnos harán prácticas (salud mental, geriatría, materno-infantil, urgencias, etc.). Se eligen los grupos de trabajo y cada uno escoge un caso que tendrá que diseñar como escenario a representar en la sala de simulación. Se realiza la lluvia de ideas sobre el caso y se marcan los objetivos de aprendizaje exponiendo de manera grupal lo que se conoce o desconoce del tema. Se realizan sugerencias sobre el diseño del escenario.

La importancia de la elaboración del problema es crucial. La configuración del conocimiento depende de la elaboración de la situación, de manera que dirige y guía todo el proceso de aprendizaje. ${ }^{25}$ Los alumnos disponen de una plantilla donde anotar las características del diseño del caso y deben asimismo disponer qué material usarán junto con el guión pormenorizado del escenario. Para la justificación de sus acciones deben realizar búsquedas en bases de datos de ciencias de la salud, obtener consejos de expertos o acudir a fuentes primarias realizando entrevistas a pacientes o a profesionales.

\section{Evaluación por competencias}

El sistema de evaluación es continuo y exige la asistencia del alumno a todas las sesiones. En la herramienta de evaluación se han incluido los siguientes aspectos:

- Informe cualitativo: Se realiza una evaluación cualitativa con los puntos fuertes, puntos que necesita mejorar y observaciones.

- Aspectos generales: Habilidades de Comunicación (con el paciente y con el equipo), priorización, reevaluación, coordinación, liderazgo y preparación de los recursos materiales.

- Informe cuantitativo: lista de chequeo ( $\mathrm{Si} / \mathrm{No}$ ) con las cinco actividades de la intervención NIC más importantes.

- Actitud: Puntualidad, iniciativa, participación, respeto, uniformidad. Cada ítem se evalúa mediante una escala Likert con 5 grados de respuesta (casi nunca, a veces, normalmente, casi siempre y siempre).

- Conocimiento: Si el alumno describe el escenario, analiza escenario, justifica con evidencias científicas, calidad de las fuentes documentales, si responde a preguntas y trabajo escrito de reflexión, apoyado en las evidencias científicas. Cada ítem se evalúa mediante una escala Likert con cinco grados de respuesta (casi nunca, a veces, normalmente, casi siempre y siempre). 


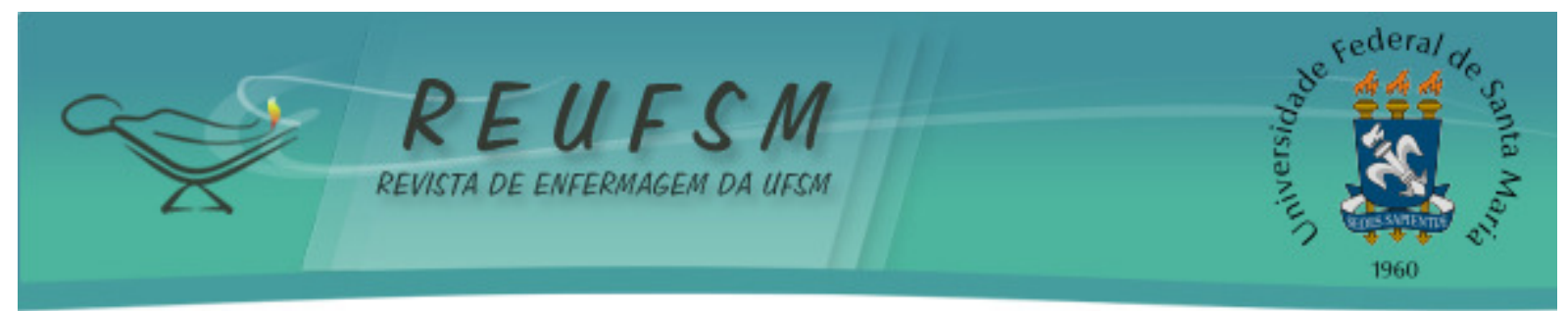

La nota final de cada escenario clínico por alumno será la suma ponderada de cada una los apartados según los siguientes porcentajes: aspectos generales (30\%), informe cuantitativo (30\%), actitud (20\%) y conocimiento $(20 \%)$.

\section{CONCLUSIONES}

Tanto el Aprendizaje basado en problemas (ABP) como la simulación clínica avanzada con simuladores a escala real son estrategias educativas de probada eficacia en la formación de los profesionales sanitarios. La unión de ambas metodologías se ha puesto en práctica con éxito en otros contextos educativos europeos. En la UCAM se ha integrado en $4^{\circ}$ de grado en enfermería dentro del Practicum clínico VI. La pertinencia de dicha fusión se traduce en una mayor implicación del alumno en la gestión de su aprendizaje y creemos que redunda en una mayor motivación que la simulación clínica por sí sola.

En este artículo se ha expuesto el modelo educativo de la simulación integrada con $\mathrm{ABP}$ en nuestro contexto. Creemos que la adaptación de este modelo es posible para su implantación en el contexto brasileño adaptando el diseño de los escenarios a la realidad asistencial de Brasil y dotando a las universidades de las infraestructuras pertinentes.

Como limitaciones podemos argumentar que en este artículo se ha presentado una experiencia educativa sin realizar una investigación ni obtener resultados más allá de la percepción subjetiva del profesorado. Se necesitan investigaciones más profundas para demostrar empíricamente que el ABP junto con la simulación clínica de alto realismo mejora objetivamente el aprendizaje y la adquisición de competencias en el grado de enfermería.

\section{REFERENCIAS}

1. López Noguero F. Metodología participativa en la enseñanza universitaria. Madrid: Nancea; 2005.

2. Cónsul Giribert M, Torrens Sígales RM. Experiencia de integración completa de un plan de estudios con el aprendizaje basado en problemas. En Echevarría Pérez P, Gómez Sánchez R, coordinadoras. Manual de aprendizaje basado en problemas: nuevas metodologías de aprendizaje en la convergencia europea. Murcia: Diego Marín; 2009. p.169-182.

3. Hospital Virtual Valdecilla (HvV). Hospital Universitario Marqués de Valdecilla [Internet].[citado 2013 sept 9]. Disponible en: http/:www.hvvaldecilla.es.

4. García Sánchez C, Alegría Capel, A. Experiencias y dificultades cinco años después de la implantación del ABP en la UCAM. En: Echevarría Pérez P, Gómez Sánchez R, coordinadoras. Manual de aprendizaje basado en problemas: nuevas metodologías de aprendizaje en la convergencia europea. Murcia: Diego Marín; 2009. p. 161-7.

5. Comisión de las Comunidades Europeas. El Marco Europeo de Cualificaciones para el aprendizaje permanente (EQF-MEC). Luxemburgo: Oficina de Publicaciones Oficiales de las Comunidades Europeas; 2009.

6. Juguera Rodríguez L, Díaz Agea JL, Pérez Lapuente ML, Leal Costa C, Rojo Rojo A, Echevarría Pérez P. La simulación clínica como herramienta pedagógica. Percepción de los alumnos de grado en enfermería en la UCAM (Universidad Católica San Antonio de Murcia). Enferm Global. 2014;13(1):175-90. 


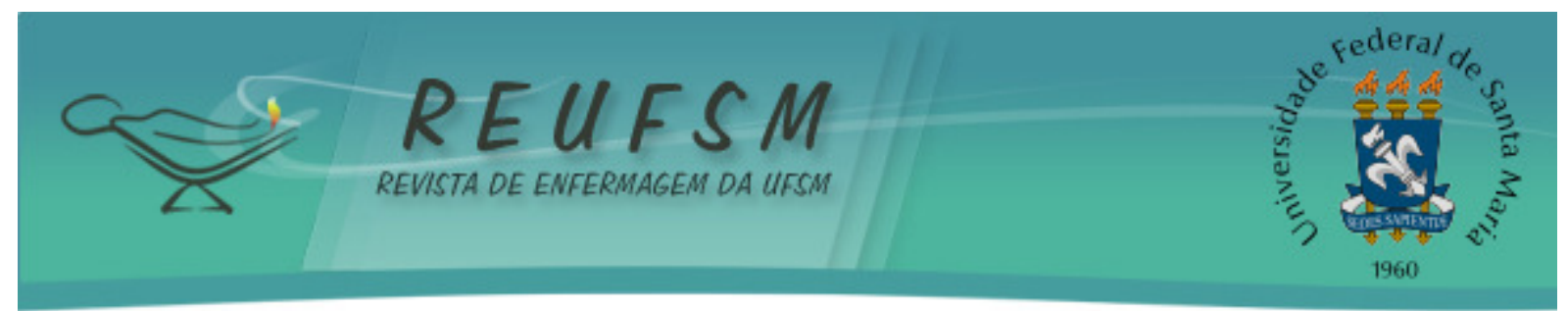

7. Murphy S, Hartigan I, Walshe N, Flynn AV, O'Brien S. Merging problem-based learning and simulation as an innovative pedagogy in nurse education. Clinical Simulation in Nursing. 2011;7(4):141-8.

8. Walshe N, O'Brien S, Murphy S, Hartigan I. Integrative learning through simulation and problem-based learning. Clinical Simulation in Nursing. 2013;9(2):47-54.

9. Clark CM, Ahten SM, Macy R. Using problem-based learning scenarios to prepare nursing students to address incivility. Clinical Simulation in Nursing. 2013;9(3):75-83.

10. Teixeira INDO, Feliz JVC. Simulação como estratégia de ensino em enfermagem: revisão de literatura. Interface (Botucatu) [Internet]. 2011 [citado 2013 mar 20];15(39):1173-84.

\section{Disponible}

http: / /www.scielo.br/scielo.php?script=sci_arttext\&pid=S1414-

32832011000400016\&lng=en\&nrm=iso. doi: http://dx.doi.org/10.1590/S141432832011005000032 .

11. Martins JCA, Mazzo A, Baptista RCN, Coutinho VRD, Godoy S, Mendes IAC, et al. A experiência clínica simulada no ensino de enfermagem: retrospectiva histórica. Acta Paul Enferm [Internet]. 2012 [citado 20 mar 2013]; 25(4):619-25. Disponible en: http://www.scielo.br/scielo.php?script=sci_arttext\&pid=S0103-

$21002012000400022 \&$ lng=en. doi: http://dx.doi.org/10.1590/S0103-21002012000400022.

12. Teixeira CRS, Kusumota L, Braga FTMM, Gaioso VP, Santos CB, Sousa VL, et al. O uso de simulador no ensino de avaliação clínica em enfermagem. Texto \& Contexto Enferm [Internet]. 2011 [citado 20 mar 2013];20 N Esp:187-93. Disponible en: http: / / www.scielo.br/scielo.php?script=sci_arttext\&pid=S0104-

07072011000500024\&lng=en\&nrm=iso. doi: http://dx.doi.org/10.1590/S010407072011000500024.

13. Santos $M C$, Leite $M C L$. A avaliação das aprendizagens na prática da simulação em enfermagem como feedback de ensino. Rev Gaúch Enferm. 2010;31(3):552-6.

14. Kolb DA. Experiential learning: experience as the source of learning and development. New Jersey: Prentice-Hall; 1984.

15. Pereira CS, Roese A, Martins AR, Pereira DB. Contribuições da educação tutorial e reorientação da formação para enfermagem: uma salada saudável. Rev Enferm UFSM [Internet]. 2013 [citado 2013 mar 20];3(21):367-73. Disponible en: http://cascavel.ufsm.br/revistas/ojs-2.2.2/index.php/reufsm/article/view/7431.

16. Branda LA. El aprendizaje basado en problemas en la formación de las ciencias de la salud. En El aprendizaje basado en problemas: una herramienta para toda la vida. Madrid: Agencia Laín Entralgo; 2004. p.17-25.

17. Hmelo-Silver CE. Problem-based learning: what and how do students learn? Educational Psychology Rewiew. 2004;16(3):199-208.

18. Gaba DM, DeAnda A. A comprehensive anestesia simulation environment: recreating the operating room for research and training. Anesthesiology. 1988;69(3):387-94.

19. Rosen MA, Salas E, Wilson KA, King HB, Salisbury M, Augenstein JS, et al. Measuring team performance in simulation-based training: adopting best practices for healthcare.

Simul Healthc. 2008;3(1):33-41. 


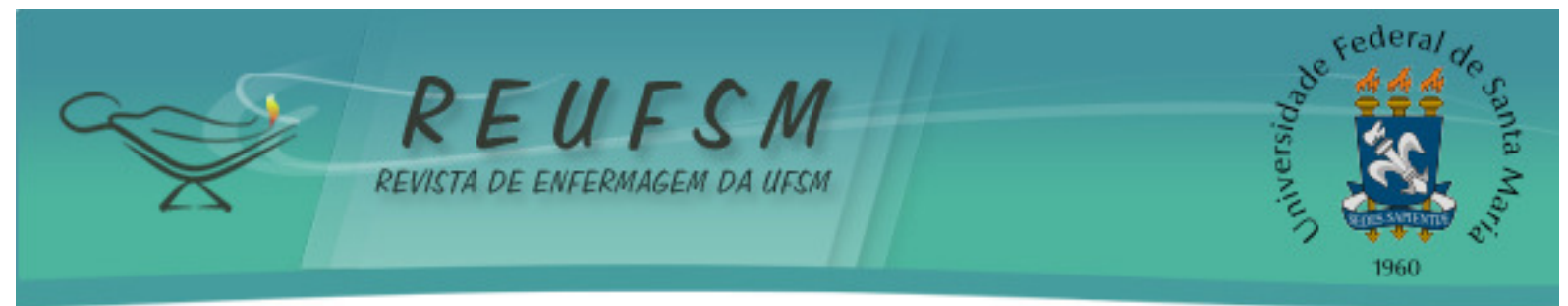

20. Herdman HT, Heath $H$, Meyer $G$, Scroggins $L$, Vassallo $B$, editores. NANDA linternational - Diagnósticos enfermeros: definiciones y clasificación 2009 - 2011. Barcelona: Elsevier; 2010.

21. Morhead S, Johnson M, Maas M, Swanson E. Clasificación de resultados de enfermería (NOC). $4^{\text {a }}$ ed. Barcelona: Elsevier; 2009.

22. Bulechek GM, Butcher HK, Dochterman JM, editores. Clasificación de Intervenciones de Enfermería (NIC). $5^{\text {a }}$ ed. Barcelona: Elsevier; 2009.

23. Ministerio de Ciencia e Innovación. Ordem CIN n²134, de 3 de julio de 2008. Establecen los requisitos para la verificación de los títulos universitarios oficiales que habiliten para el ejercicio de la profesión de enfermero [Internet]. BOE; 2008 jul 19 [citado 2014 mar 20]. Núm. 174, p. 31680-3. Disponible en: http://www.uma.es/ordenac/docs/News/RequisitosEnfermero.pdf.

24. Díaz Cuenca A, Díaz Agea JL. El papel del tutor de ABP. En: Echevarría Pérez P, Gómez Sánchez R, coordinadoras. Manual de aprendizaje basado en problemas. nuevas metodologías de aprendizaje en la convergencia europea. Murcia, Diego Marín; 2009. p. 77-89.

25. Font Rivas A. Líneas maestras del aprendizaje por problemas. El reto del Espacio Europeo de Educación Superior. Revista Interuniversitaria de Formación del profesorado. 2004;18(1):85-6.

Data de recebimento: $03 / 10 / 2013$

Data de aceite: 19/08/2014

Contato com autor responsável: José Luis Díaz Agea

Endereço postal: Universidad Católica de Murcia (UCAM). Laboratorio de Simulación Clínica. Facultad de Enfermería. Campus de los Jerónimos s/n. 30107. Guadalupe (Murcia). España. Tfno. (+34) 968278183.

E-mail: jluis@ucam.edu 